\title{
Combining a Novel Computer Vision Sensor with a Cleaning Robot to Achieve Autonomous Pig House Cleaning
}

\author{
Andersen, Nils Axel; Braithwaite, lan David; Blanke, Mogens; Sørensen, Torben
}

Published in:

Proceedings of the 44th IEEE Conference on Decision and Control, and European Control Conference

Link to article, DOI:

10.1109/CDC.2005.1583511

Publication date:

2005

Document Version

Publisher's PDF, also known as Version of record

Link back to DTU Orbit

Citation (APA):

Andersen, N. A., Braithwaite, I. D., Blanke, M., \& Sørensen, T. (2005). Combining a Novel Computer Vision Sensor with a Cleaning Robot to Achieve Autonomous Pig House Cleaning. In Proceedings of the 44th IEEE Conference on Decision and Control, and European Control Conference (pp. 8331-8336). IEEE. https://doi.org/10.1109/CDC.2005.1583511

\section{General rights}

Copyright and moral rights for the publications made accessible in the public portal are retained by the authors and/or other copyright owners and it is a condition of accessing publications that users recognise and abide by the legal requirements associated with these rights.

- Users may download and print one copy of any publication from the public portal for the purpose of private study or research.

- You may not further distribute the material or use it for any profit-making activity or commercial gain

- You may freely distribute the URL identifying the publication in the public portal 


\title{
Combining a Novel Computer Vision Sensor with a Cleaning Robot to Achieve Autonomous Pig House Cleaning
}

\author{
Nils Axel Andersen ${ }^{1}$, Ian D. Braithwaite ${ }^{1}$, Mogens Blanke ${ }^{1}$ and Torben Sørensen ${ }^{2}$
}

\begin{abstract}
Cleaning of livestock buildings is the single most health-threatening task in the agricultural industry and a transition to robot-based cleaning would be instrumental to improving working conditions for employees. Present cleaning robots fall short on cleanness quality, as they cannot perform condition based cleaning. This paper describes how a novel sensor, developed for the purpose, and algorithms for classification and learning are combined with a commercial robot to obtain an autonomous system which meets the necessary quality attributes. These include features to make selective cleaning where dirty areas are detected, that operator assistance is called only when cleanness hypothesis cannot be made with confidence. The paper describes the design of the system where learning from experience maps and operator instructions are combined to obtain a smart and autonomous cleaning robot.
\end{abstract}

\section{INTRODUCTION}

$\mathrm{M}$ ANUAL cleaning of livestock buildings, using highpressure water technology, is one of the most tedious and health threatening tasks which is conducted by human labor. The cleaning process itself contributes to deterioration of the working environment due to stirring up dirt, microorganisms and water, which is inhaled by the operator. Consequently, it is essential to improve the working conditions for personnel who are performing today's cleaning of livestock buildings. Societies' concern about food safety and livestock welfare are other essential issues.

Ongoing research in Europe includes development of cleaning robots, of which few are commercialized. Investigations of current devices have shown that robot performance is poor regarding effectiveness and utilization of detergent and water. The water consumption for robotic cleaning is up to $40 \%$ higher than what is used for manual

Manuscript received March 7, 2005. This research was carried out in the ISAC project supported by the Danish Ministry of Science, Technology and Development and by the Minstry of Food, Agriculture and Fisheries under the sustainable technology in agriculture programme, grant number 205301-0021

The ISAC project is carried out by the departments Ørsted-DTU, IMM and MEK at the Technical University of Denmark in collaboration with Research Centre Bygholm at the Danish Institute of Agricultural Sciences, the Pig Producers Association and the companies ALTO and GERNI.

Nils A. Andersen, Ian D. Braithwaite and Mogens Blanke are with section of Automation at Ørsted•DTU, Building 326, Technical University of Denmark DK2800 Kgs. Lyngby, Denmark, naa, idb, mb@oersted.dtu.dk

Torben Sørensen is with the Department of Mechanical Engineering, Building 404 Technical University of Denmark, DK2800 Kgs. Lyngby, Denmark, ts@mek.dtu.dk cleaning [1] Furthermore; subsequent manual cleaning is needed [2] as present robots are unable to detect the cleanness of surfaces

Robot based cleaning being developed for household and similar mass markets exist in different forms [2]. While research prototypes of industrial grade cleaning devices have emerged [3], none are yet based on sensor input to guide the cleaning process. While computer vision is widely applied in automation and surveillance, classification of a pig pen surface as clean is difficult due to inhomogeneity, aging, wear and the risk of misclassification originating from these factors. These obstacles have recently been solved by a dedicated sensor system (patent pending) that can obtain high quality cleanness reports [4].

This paper shows how cleanness maps from the new sensor are combined with cleaning history and past cleanness inspections to form an experience map from which present and future cleaning sequences are planned. It is discussed how operator advice is incorporated and how learning is achieved. The software architecture for the autonomous system is presented and use-cases illustrate how autonomous cleaning is conducted. The system presented is the first to implement autonomous pig house cleaning based on cleanness inspection.

\section{Cleaning REQUiREMENTS}

In modern livestock production, proper cleaning of floors and housing equipment is essential to maintain livestock health, product quality and food safety. Therefore batch production widely used in large-scale pig production involves an entire section being emptied and cleaned before a new batch of young livestock is entered. The cleaning is presently done manually using high-pressure water cleaners.

Two robot cleaners were recently introduced [5] for pig house cleaning, but the use is still scarce. Both robots are programmed to run specified cleaning patterns moving their high-pressure water jets. Neither have sensors to determine cleanness. This leads to a tradeoff between over-cleaning with excessive water usage and cleaning left for manual finishing of the job. In [5] a user of one of the robots states that only $80 \%$ of the cleaning is managed by the robot.

The robot system described in this paper is expected to overcome this problem by using a newly developed cleanness sensor for controlling the cleaning process. 


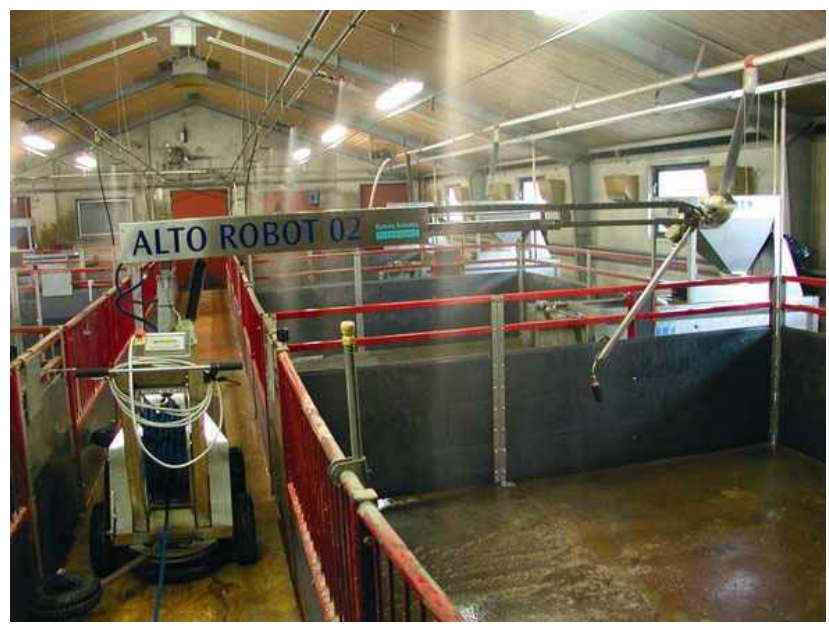

Fig. 2 Pighouse cleaning robot in action using high-pressure water to remove manure from a pig pen.

\section{Cleanness Sensor}

In order to detect the cleanness of surfaces before, during and after cleaning, our robot is equipped with a non-invasive cleanness sensor. Based on computer vision techniques and a dedicated lighting system, on which a patent is pending, the sensor can provide a cleanness measure over a relatively large area quickly and accurately. Mounting the sensor on the robot together with the cleaning system, it is assured that all areas washable can also be inspected.

\section{A. Sensor Operation}

The robot cleanness sensor consists of a computer vision camera, together with an illumination system and integrated control. High-power light emitting diodes are used to generate a sequence of specific wavelengths, illuminating the surface in turn. A sequence of monochrome pictures are captured for each area under inspection. Each group of pictures is then analyzed using a multivariable statistical method described in [4], resulting in a pixel-by-pixel description of the cleanness of the surface in view.

In this application, dirt particles down to $2 \mathrm{~mm}$ in diameter must be recognized, leading to a natural pixel size of equal or slightly smaller dimension. A computer vision camera with $1 / 4$ million pixels can cover an area of $0.5 \mathrm{~m}$ by $0.5 \mathrm{~m}$ with pixels corresponding to millimeter squares.

The image processing system receives multiple images, or a 'multi-spectral' image, as input. After processing, a corresponding cleanness map is produced, where individual pixels are labeled as either "clean", "dirty" or "undetected". The sensor gives a "clean" classification where, with a degree of certainty, the background material, for example concrete, is detected. The class "dirty" is given, correspondingly, where pig manure is detected. If neither classification is sufficiently certain, "undetected" is indicated: the camera might not be properly positioned for a clear view of a surface, an unknown object might be in view or material wear and damage may be beyond what the sensor can reliably work with.

Fig. 1 shows two test surfaces, one clean and one largely dirty, together with the corresponding outputs from the image processing. In the images showing the classification, clean areas are marked with light color and dirty areas with dark color. The dirty concrete is largely classified as dirty and the clean concrete largely as clean. A closer look at the dirty image reveals that most of the pixels classified as clean actually are consistent with areas in the real picture that could actually be clean so the number of misclassifications is small. On the other hand looking at the picture of the clean concrete it is clear that parts of the dark spot at the upper edge of the image, and one other spec are misclassified as dirty. These artifacts cannot be removed by plain image processing but learning needs to be employed.

\section{B. Cleanness Map}

Millimeter cleanness data is important to ensure the

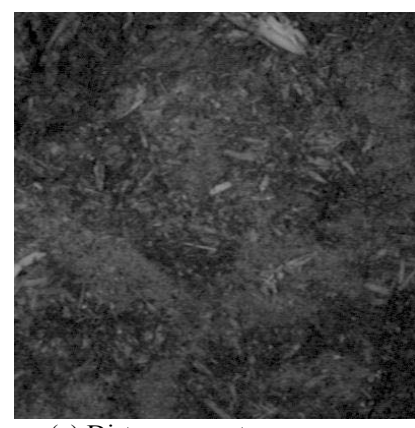

(a) Dirty concreate

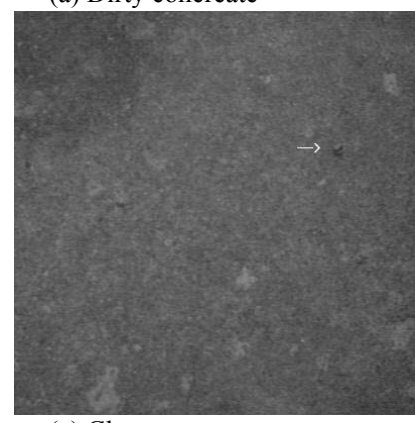

(c) Clean concreate

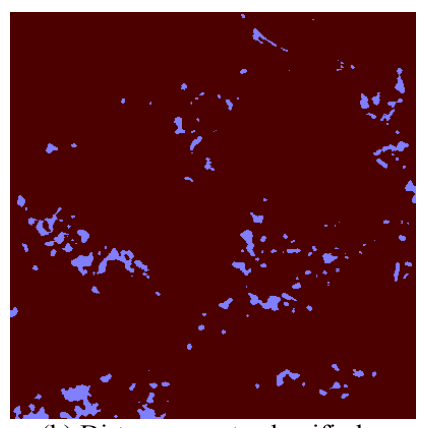

(b) Dirty concreate classified

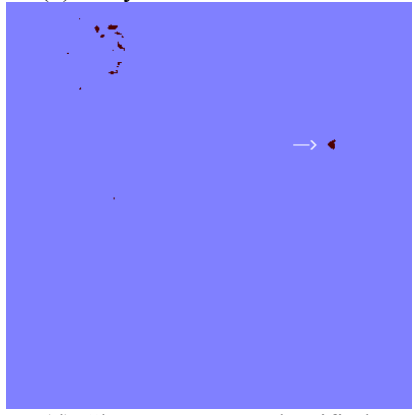

(d) Clean concreate classified
Fig. 1 Classification results from cleanness sensor. Real image to the left classification to the right. In the classification images light colour means clean and dark colour dirty. The arrows show a spec classified as dirty due to age effects in the floor. Misclassification of such objects is avoided by learning.

cleaning required in the application, but too fine grained for robot planning and control. Thus, a cleanness map with a larger resolution is generated from the pixel-level data, indicating the level of cleanness in larger areas across the pig pen. Fig. 3 shows the walls and the floor of a single pig pen folded out to a 2-d figure. If several pixels are classified as undetectable the whole square is marked as undetectable 
using a small circle. The figure exemplifies a cleanness map after a first raw cleaning of a pen. The map provides the position of remaining dirt and is used by the system to plan the next cleaning pass. Another use of the map is to document the cleaning results after cleaning is finished.

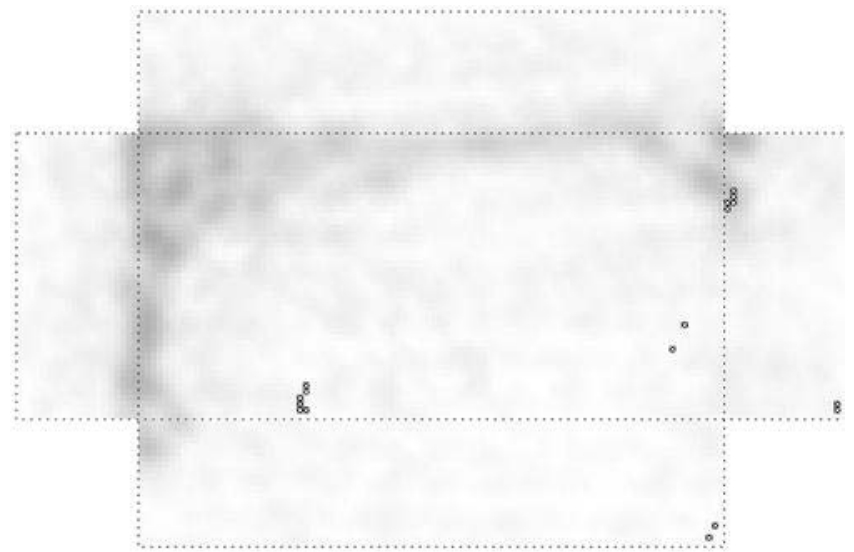

Fig. 3 Cleanness map showing degree of cleanness. Greyscale indicates degree of cleanness, small circles mark undetectable.

\section{FUnCTIONAL DECOMPOSITION}

A hierarchical break down of the cleaning process is described below and illustrated in Fig. 4. This description is the basis for subsequent design of the software of the autonomous robot.

\section{A. Section Service.}

The highest level task specification relevant for a pig house cleaning robot is cleaning and/or inspection of a section with $12-20$ pig pens. This level is called the section service level. At this level, a plan is generated that decides

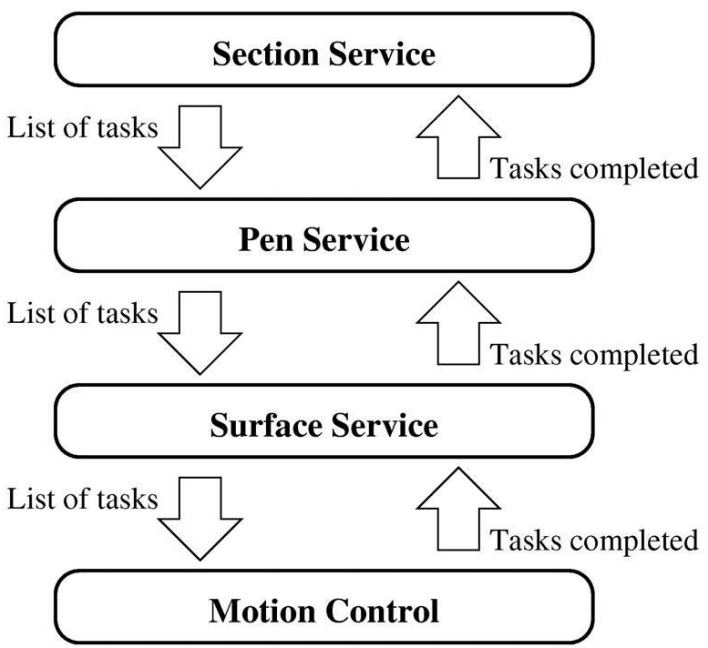

Fig. 4 Hierarchical break down of the cleaning process

which pig pens should be serviced, what service each pen should receive and the sequence of pens to be serviced. This plan is generated by the operator.

\section{B. Pen Service}

The next level is the pen service level. At this level, plans are generated for cleaning and inspection of a single pen. Information about geometry, obstacles such as feeding devices, piping and material is used to divide the task into the basic task of servicing a surface or a complex object e.g. a feeding device. Typical subtasks are cleaning of wall, cleaning of floor, cleaning of specified area and remove specks detected on a surface. After dividing the task into subtasks, the sequence of subtasks is decided. This is important as dirt cleaning one surface may affect already cleaned surfaces. The plans at this level can be user assisted to enable the user to instruct the robot system or have full control of certain sequences, as will be illustrated in the use cases below. Unsupervised generation of plans are done based on interpretation of the pen geometry map, the summary experience map and the current condition map.

Data stored by the system include

- Geometry map with specification of materials and landmarks

- Current cleanness map that holds the information on the current cleanness of all surfaces of the pen.

- Experience map is an extract with relevant features from previous inspections of the particular pen, cleaning history and user assisted characterization.

\section{Surface Service}

The surface service level is responsible for generating proper trajectories for the required service. The two required services are cleaning and inspection. The trajectories for cleaning are generated to obtain coverage of the surface while keeping the high-pressure water-cleaning tool at a specified distance and angle to the surface to be cleaned. Meng et.al [10] shows how the effectiveness of cleaning depends on distance and pressure. The planner also directs the water jet such that removed manure does not contaminate already cleaned areas.

Trajectories for inspection are generated to ensure full coverage with certain overlap between pictures and inclusion of specified landmarks (corners etc) in some of the pictures. Both types of trajectories account for obstacles and keep a safe distance of the robot and the arm. Output from the surface service layer is a list of elementary robot or camera movements necessary to execute the trajectory.

\section{Motion control layer}

In the motion control layer, the trajectories are converted to actual robot and camera command sequences.

\section{INSPECTION AND CLEANING}

\section{A. Cleaning Strategy Using Sensor Feedback.}

Optimal cleaning would probably require a continuous measurement of the cleanness in the active cleaning area. Due to the water and dirt spray generated by the cleaning process this will not be possible with the vision-based sensor 
used in this project. Instead a strategy that alternates between inspection and cleaning will be used.

The best strategy is expected to start with an open loop cleaning that based on experience will clean most of the pen. After this the pen is inspected and the result of the inspection is used to guide the next cleaning process. This is continued until the inspection results are satisfactory. The cleaning process is finished with a full inspection.

\section{B. Cleanness Report.}

After a cleaning and inspection task is finished a report of the inspection is available. The cleanness is presented in a map of each pig pen showing the distribution of remaining dirt using color to represent degree of cleanness.

Key numbers for cleanness, water and time consumption are given as well as warnings about areas that might need human inspection due to persistent classification as dirty.

\section{Planning of Trajectories.}

The robot has two different tasks to do; one is to move the camera-sensor for inspection of cleanness and the other to move the high-pressure water gun for cleaning. Trajectories are needed to control the tool positions and speeds for both of these tasks. A trajectory is a description of a path in space including time/speed information.

The target robot is basically a robotic manipulator arm with five degrees of freedom mounted on wheels that allows it to drive forwards and backwards. The arm has three joints for positioning the camera and two that can orient the water gun head as shown in Fig. 5. The camera has pan and tilt in addition.

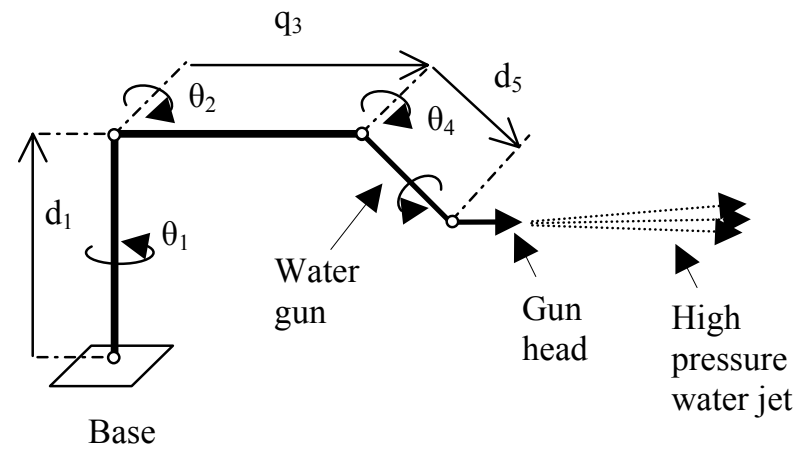

Fig. 5 Kinematics of the Alto (Ramsta Robotics) target robot.

The target robot is constructed by the manufacturer to execute only one motion at a time and there is a short delay before moving a new joint. With only one joint moved at a time, the trajectory needs to be planned to minimize the number of intermediate configurations and tool patterns. This task is done by the trajectory generator module.

\section{SOFtware Architecture}

A modular, component-based software architecture has been designed to implement the robot cleaning system, as illustrated in figure 6 . Rectangular boxes in the figure represent software components, which are distributed amongst the computer devices in the system. Although the component based architecture can in itself have advantages, it is also a necessity because at the very least the robot controller, the sensor system and the user interface are located on different devices.

Communication between components is carried by TCP/IP, either internally for components located on the same device, or over a dedicated Ethernet link for components on different devices. The high-level protocol messages are encoded as XML. The graphical user interface (GUI) employs a wireless network. The display device is a standard Web browser, and the protocol is HTTP.

From top to bottom, the individual components are responsible for the following.

1) Graphical User Interface

This component is an embedded Web server, which generates dynamic pages as a result of queries sent to the other components in the system. In particular, it interacts with the Planner, in order to present new jobs to the system and to monitor progress of the current job. Additionally, information about the pig house topology can reviewed and modified.

The GUI also interacts with the Mapper, in order to find more detailed information about the results of an inspection. Cleanness reports can be viewed showing the results for individual elements in the pig house, and an experience map can be updated with new information about the individual elements. Links to the other components in the system are provided such that the user can monitor progress further down in the system. Video images from the vision sensor can be viewed, for example, if a particular artifact needs be remotely inspected.

\section{2) Planner}

This component handles the highest level of task control in the software system. Information about the pig house at the topological level is manipulated, including elements such as walls and feeding devices. Knowledge stored about the building sections allows the planner to translate user commands to a list of tasks on the individual elements. Washing sequences are also planned at this level based on feedback from the cleanness and experience maps.

3) Mapper

This component handles the system's 3D model: the robot and the environment in which it operates. The Mapper can plan trajectories for the robot within the pig house section.

Information about the positions and orientations of the individual elements in the building are stored, based on an initial model, and potentially modified by measurements from the system's own sensors. Cleanness information from the sensor is also stored for each element in the form of a cleanness map as this data becomes available. In this way, subsequent trajectory planning can take account of previous measurements. While the cleanness map represents shortterm knowledge about the state of building materials, an 
experience map contains longer-term knowledge. Permanent

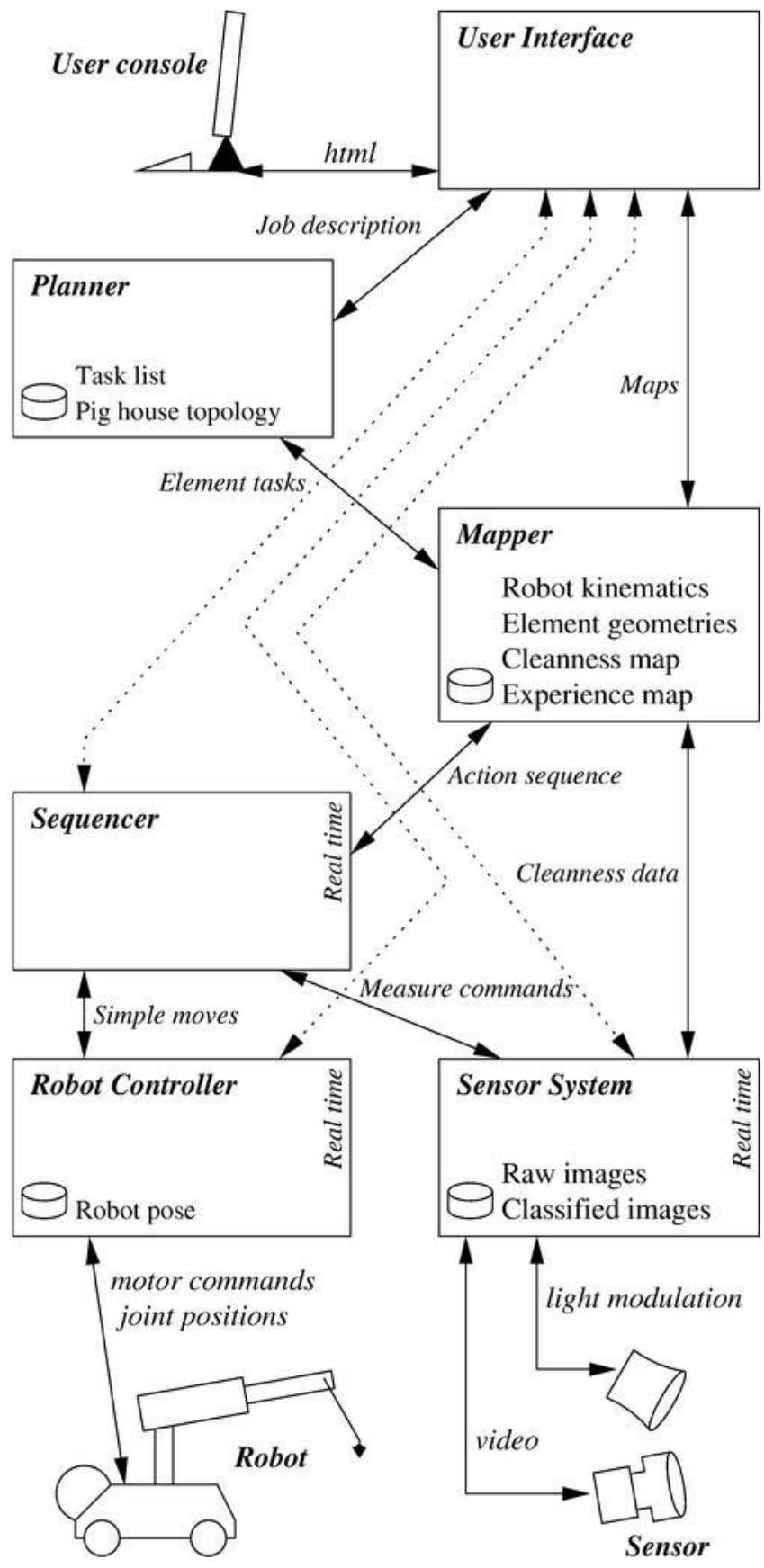

Fig. 6 Component based software architecture

damage that can not be improved by more cleaning, but in any case can appear as dirt can also be recorded and taken into account when planning future detailed cleaning.

Information stored about the individual elements is based on a grid covering the particular element. The grid size is chosen to suit the properties of the cleaning process.

4) Sequencer

This component is the first component with hard real-time requirements. It receives sequences of robot moves, interleaved with sensor commands, from the Mapper. Its task is to coordinate the Robot Controller and the Sensor System such that timing constraints between the two are upheld.

Real-time behavior is necessary, for example, because the position and orientation of the sensor must be known in order to successfully interpret the sensor output. Thus, time must be provided for the robot position to stabilize after a move, and the sensor must be held steady for the duration of the measurement cycle.

\section{5) Robot Controller}

This component receives simple movement commands from the Sequencer and communicates directly with the robot hardware to carry out the requested movement. Sensors in the robot joints enable the controller to track actual robot position. Hard real-time (HRT) behavior is necessary for the robot hardware interaction.

\section{6) Sensor System}

This HRT component communicates with the video camera and illumination devices. Cleanness measurements are made by capturing a series of images with a lighting sequence provided by the system. Raw images are stored together with results provided by the classifier/image analysis algorithms. Summary results are made available to the Mapper in a form with individual pixels collected together into larger tiles for use in planning.

\section{AUTONOMOUS SUPERVISION AND LEARNING}

The recent history of cleaning and the results of camera inspections are available in the experience map. The detailed information in the most recent cleanness map is used by the planner module, first in the initial planning of overall cleaning of the dirty pen and later to make a shortlist of areas that need intensive cleaning.

\section{A. Learning}

When particular areas of the pen become aged, color profiles of surfaces will change. Daily use may also cause small cracks in the surface that need special attention as dirt will adhere better to such spots. When repeated observations indicate permanent changes of an area, this is marked up for manual assistance. Manual inspection is rapidly done, using the coordinates and the picture shown at the graphical user interface. Having inspected an area, the human's classification is entered, and this information is used subsequently by the autonomous classifier and planner. A use case is presented as illustration.

\section{B. Use Case}

When a pen is first washed, the distribution of dirt is unknown. A washing scheme called 'first' is activated by the planner based on pen geometry and desired flow direction.

After the first cleaning, a total pen inspection is made. The cleanness map shows where dedicated washing is needed. A washing scheme called 'intensive' is then used at selected areas. The planner commands spray angle and 


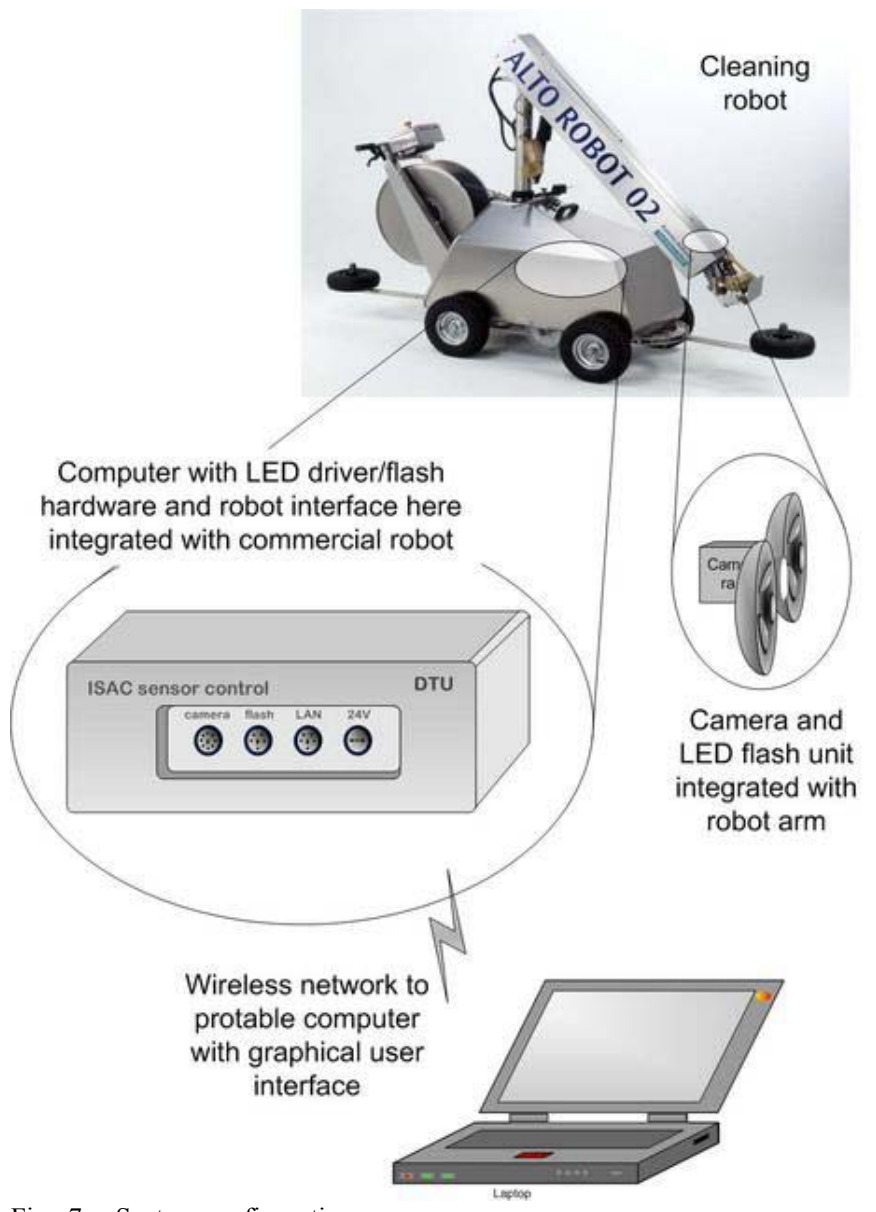

Fig. 7 System configuration.

distance as needed. A second inspection shows the results of the intensive cleaning. Using the experience map and the immediate cleaning history data, an active learning algorithm makes a hypothesis about the particular remains and requests a third washing scheme, with parameters that reflect the latest hypothesis about the remains. After executing the third local high-pressure washing, a final inspection is made of the entire pen. If specks remain classified as dirty, the user is prompted to decide on the character. An assisted learning algorithm then updates the experience map. Future actions could include: intensive cleaning is incorporated already in the first washing scheme for the area; categorization algorithm parameters are altered for the relevant area; cleanness report calling on physical maintenance.

\section{System PRototype}

An experimental prototype of the system is shown in Fig. 7. The main components are the target robot platform from ALTO (a Ramsta Robotics development) with the cleanness sensor added. The cleanness sensor consists of a camera and a dedicated lighting system interfaced to an imbedded PC running the Slackware GNU Linux operating system. The robot is interfaced to a second computer unit, the robot PC, via a galvanic isolated interface. The robot $\mathrm{PC}$ is running RTAI real-time Linux. The two computers are connected via cable and to a laptop via a wireless Ethernet. The laptop runs the GUI with the user well away from the pig house.

\section{CONCLUSION}

A design of a sensor based cleaning robot was presented. The design improves the cleaning performance due to the sensor guided cleaning procedure assisted by active or assisted learning. The software architecture for the combined sensor and robot system was presented and implementation issues discussed. The main contribution is a novel application of autonomous robotics that will help reduce human presence in a hostile working environment.

\section{ACKNOWLEDGMENTS}

The authors acknowledge the work done by co-workers and students: S. A. Rosenkær, E. Millan and R. Galeazzi. The test robot was kindly provided by ALTO A/S.

\section{REFERENCES}

[1] J. S. Strøm, G. Zhang and M. Blanke: "Intelligent sensor for autonomous cleaning in livestock buildings - a challenge in bioenvironmental engineering". Proc. IFBBE 2003, Beijing, pp 283288.

[2] P. Fiorini and E. Prassler: "Cleaning and household robots - a technology survey". Autonomous Robotics 9 pp 227-235, 2000.

[3] E. Prassler, A. Ritter, C. Schaeffer and P. Fiorini: "A short history of cleaning robots". Autonomous Robotics 9: 211-226, 2000

[4] I. D. Braithwaite, M. Blanke, G. Zhang and J. M. Carstensen: "Design of a Vision-based Sensor for Autonomous Pighouse Cleaning”. Technical University of Denmark, Kgs. Lyngby, Denmark, 2004. EURASIP Journal of Applied Signal Processing, 2005. In print.

[5] A. Evans: "Simple to operate but technically advanced", Pig Progress, 20 (2): 22-24, 2004

[6] G. Smith and C. Hofner: "An advanced planning and navigation approach for autonomous cleaning robot operations". Proc. 1998 IEEE/RSI Int. Conference on Intelligent Robots and Systems, Victoria BC, Canada Oct. 1998 pp 1230-1235.

[7] H. Choset.: "Coverage for robotics - a survey of recent results". Annals of Mathematics and Artificial Intelligence 31: 113-126, 2001

[8] J. M. Hickey and L. E. Hise: "Color image processing and vision system for an automated laser paint stripping system". Intelligent Robotics and Computer Vision XIII: vol 2353, pp 503-512, 1994.

[9] M. King, and B. Zhu: "Gaming strategies". In Path Planning to the West, vol. II, S. Tang and M. King, Eds. Xian Jiaoda Press, 1998 pp. 158-176.

[10] P. Meng,, M. C. Leu, E. S. Geskin and L. Tismenetskiy: "Cleaning with high-pressure directed waterjets". In Proc. of the Japan-USA Symposium on Flexible Automation - 1996 vol 2: 1131-1138. ASME. 1996.

[11] M. Simoncelli, G. Zunino and H. I. Christensen: "Autonomous pool cleaning: self-localization and autonomous navigation for cleaning". Autonomous Robotics 9, 2000, pp 261-270.

[12] Simpson, H. Dumb Robots, $3^{\text {rd }}$ ed., Springfield UOS Press, 2004, pp.69. 